\title{
The Jacobi diffusion process as a neuronal model
}

Giuseppe D’Onofrio, Massimiliano Tamborrino, and Petr Lansky

Citation: Chaos 28, 103119 (2018); doi: 10.1063/1.5051494

View online: https://doi.org/10.1063/1.5051494

View Table of Contents: http://aip.scitation.org/toc/cha/28/10

Published by the American Institute of Physics

\section{Don't let your writing} keep you from getting published!

AlP Author Services

Learn more today! 


\title{
The Jacobi diffusion process as a neuronal model
}

\author{
Giuseppe D’Onofrio, ${ }^{1, a)}$ Massimiliano Tamborrino, ${ }^{2, b)}$ and Petr Lansky ${ }^{1, c)}$ \\ ${ }^{1}$ Institute of Physiology of the Czech Academy of Sciences, Videnska 1083, 14220 Prague 4, Czech Republic \\ ${ }^{2}$ Johannes Kepler University Linz, Institute for Stochastics Altenbergerstraße 69, 4040 Linz, Austria
}

(Received 9 August 2018; accepted 1 October 2018; published online 24 October 2018)

\begin{abstract}
The Jacobi process is a stochastic diffusion characterized by a linear drift and a special form of multiplicative noise which keeps the process confined between two boundaries. One example of such a process can be obtained as the diffusion limit of the Stein's model of membrane depolarization which includes both excitatory and inhibitory reversal potentials. The reversal potentials create the two boundaries between which the process is confined. Solving the first-passage-time problem for the Jacobi process, we found closed-form expressions for mean, variance, and third moment that are easy to implement numerically. The first two moments are used here to determine the role played by the parameters of the neuronal model; namely, the effect of multiplicative noise on the output of the Jacobi neuronal model with input-dependent parameters is examined in detail and compared with the properties of the generic Jacobi diffusion. It appears that the dependence of the model parameters on the rate of inhibition turns out to be of primary importance to observe a change in the slope of the response curves. This dependence also affects the variability of the output as reflected by the coefficient of variation. It often takes values larger than one, and it is not always a monotonic function in dependency on the rate of excitation. Published by AIP Publishing. https://doi.org/10. 1063/1.5051494
\end{abstract}

Diffusion processes with multiplicative noise are able to model the changes in the membrane depolarization between two consecutive spikes of a single neuron. Among them, the most commonly used models consider either no state-space boundaries or only a lower boundary. Nevertheless, the upper boundary also modifies the properties of the model due to the multiplicativity of the noise. Here, we consider the Jacobi diffusion process that is able to include, in its neuronal interpretation, both excitatory and inhibitory reversal potentials (upper and lower boundary, respectively). In the Jacobi neuronal model, the involved parameters are input-dependent and this, together with the form of the multiplicative noise, affects the output of the model. The response of the neuron is studied focusing on the behavior of the first-passage time through the firing threshold, identified with the dynamics of spike generation. Computationally easy expressions of the first two moments of the first-passage time for the Jacobi process are derived and implemented to study the firing rate and variability of the inter-spike intervals. The third moment is given as a tool for the parameter estimation. All the results concerning the first-passage-time problem are general and can be used outside the framework of computational neuroscience.

\section{INTRODUCTION}

In the models where noise is assigned a casual importance, it is often assumed to be a source of inefficiency and unpredictability. However, there exists a large class of

\footnotetext{
a)Electronic mail: giuseppe.donofrio@unito.it

b)Electronic mail: massimiliano.tamborrino@jku.at

c)Electronic mail: lansky@biomed.cas.cz
}

phenomena for which the noise is of a primary importance or even a part of the signal itself. Mathematical models in neuroscience are one of the most prominent examples in this direction. The celebrated model of Lapicque, republished and discussed at its centennial anniversary, ${ }^{1,2}$ was deterministic, but its numerous generalizations took into account the inputoutput variability of the neurons in the networks and became intrinsically stochastic. From a biophysical point of view, the models of a single neuron reflect the electrical properties of its membrane. Such circuit models can be written in terms of differential equations for the membrane voltage. To reduce their mathematical complexity, integrate-and-fire types of the models have been derived., ${ }^{3,4}$ These models aim to describe the dynamics of interspike intervals, and they are based on a one-dimensional representation of the time evolution of the neuronal membrane depolarization.

One class of stochastic models which can be classified as a generalization of the Lapicque model with restricted state space appeared in the 1960 s. Stein ${ }^{5}$ and Johannesma ${ }^{6}$ introduced models with conductance changes caused by excitatory and inhibitory inputs. The state space of the depolarization was limited between two boundaries, the inhibitory and the excitatory reversal potentials. Later, this type of models with multiplicative noise started to be extensively investigated. ${ }^{7-11}$ These studies often employed the diffusion limits of the originally discontinuous models to improve their tractability. Among them, the most commonly investigated model considers the lower boundary only, and with its specific form of the diffusion coefficient, it can be identified as the Feller process (in biology), also known as Cox-Ingersoll-Ross (in finance) or square-root process (in mathematics). Nevertheless, there seems to be no strong mathematical argument for the selection of a specific form of the diffusion coefficient which controls the state space of the depolarization. Thus, several other 
different variants have been investigated. ${ }^{11,12}$ The emphasis on the lower boundary, the inhibitory reversal potential, appears probably because the role of the upper boundary, the excitatory reversal potential, seems to be blurred by imposing below it a firing threshold. However, despite the existence of the threshold, the upper boundary modifies the properties of the model due to the multiplicativity of the noise.

The studies in which both reversal potentials are considered are more rare, ${ }^{13-16}$ and the present article is devoted to such a model. Here, the effect of multiplicative noise on the output of the Jacobi neuronal model with dependent parameters is examined in detail. After introducing the Jacobi diffusion process and describing the firing mechanism which is based on the solution of the first-passage-time problem, the expressions for the first three moments are presented. Up to this point, the results are independent of the application in neuroscience. Further, intrinsic dependency of the parameters as it follows from the biophysical model is presented, and results for such a model are deduced and interpreted.

\section{THE PEARSON DIFFUSION MODELS}

One-dimensional stochastic differential equations play a key role in the description of fluctuating phenomena belonging to different fields of applications as physics, biology, neuroscience, finance, and others. ${ }^{17}$ In particular, the class of models with a linear drift and driven by a Wiener process is widely used for its mathematical tractability and flexibility. These models are described by a stochastic differential equation of the following type

$$
d Y_{t}=\left(-\alpha Y_{t}+\beta\right) d t+\Sigma\left(Y_{t}\right) d W_{t}, \quad Y_{0}=y_{0},
$$

where $\alpha>0, \beta \in \mathbb{R}, W=\left\{W_{t}\right\}_{t \geq 0}$ is a standard Wiener process and $Y_{0}=y_{0}$ is the initial condition. The diffusion coefficient $\Sigma\left(Y_{t}\right)>0$ determines the amplitude of the noise, and, according to its dependence on $Y_{t}$, it characterizes the solution of Eq. (1). If $\Sigma\left(Y_{t}\right)=\sqrt{a Y_{t}^{2}+b Y_{t}+c}$ for $a, b, c \in \mathbb{R}$, the solution of Eq. (1) is called Pearson diffusion process. ${ }^{18} \mathrm{~A}$ wide range of well-known processes belongs to this class. The solution of Eq. (1) is an Ornstein-Uhlenbeck process for $\Sigma\left(Y_{t}\right)=\sigma>$ 0 constant (i.e., $a=b=0, c=\sigma$ ), a Feller process (also known as Cox-Ingersoll-Ross model or square-root process) for $\Sigma\left(Y_{t}\right)=\sigma_{F} \sqrt{Y_{t}}, \sigma_{F}>0$ (i.e., $b=\sigma_{F}^{2}, a=c=0$ ), an inhomogeneous geometric Brownian motion for $\Sigma\left(Y_{t}\right)=\sigma_{G} Y_{t}, \sigma_{G}>0$ (i.e., $a=\sigma_{G}^{2}, b=c=0$ ), and a Jacobi diffusion (or Wright-Fisher diffusion process) for $\Sigma\left(Y_{t}\right)=\sigma_{J} \sqrt{Y_{t}\left(1-Y_{t}\right)}$ (i.e., $\left.a=-\sigma_{J}^{2}, b=\sigma_{J}^{2}, c=0\right)$. Throughout this work, we will focus on this last process.

The values taken by model (1), i.e., its state space, are in the interval $-\infty \leq B_{1}<y_{0}<B_{2} \leq+\infty$. The endpoints $B_{1}$ and $B_{2}$ can or cannot be reached in a finite time depending on the underlying parameter conditions. According to the Feller's classification of boundaries, ${ }^{19} B_{1}$ is an entrance boundary if it cannot be reached by $Y_{t}$ in finite time, and there is no probability flow to the outside of the interval $\left(B_{1},+\infty\right)$, that is, the process stays in $\left[B_{1},+\infty\right)$ with probability 1 . Vice versa, $B_{1}$ is an exit boundary if the process can attain it, but then it cannot return into $\left(B_{1},+\infty\right)$. The latter situation is not suitable for our modeling purposes, and thus, it is not considered here. An analogous classification for $B_{2}$ can be stated.

We denote the transition probability density function (pdf) of the diffusion process (1) by $f(y, t \mid z, \tau)$. If $\mathcal{W}(y):=$ $\lim _{t \rightarrow \infty} f\left(y, t \mid y_{0}, 0\right)$ exists independently on $y_{0}$, we say that the process $Y_{t}$ admits a stationary distribution $\mathcal{W}(y)$. Independently on the choice of $\Sigma\left(Y_{t}\right)$, the mean of $Y_{t}$ in (1) is given by

$$
\mathbb{E}\left[Y_{t} \mid Y_{0}=y_{0}\right]=\frac{\beta}{\alpha}+\left(y_{0}-\frac{\beta}{\alpha}\right) e^{-\alpha t},
$$

and the asymptotic mean by

$$
\lim _{t \rightarrow+\infty} \mathbb{E}\left[Y_{t} \mid Y_{0}=y_{0}\right]:=\mathbb{E}\left[Y_{\infty}\right]=\frac{\beta}{\alpha},
$$

while higher moments of $Y_{t}$ depend on the function $\Sigma\left(Y_{t}\right)$ in (1).

Let the process $Y_{t}$ evolve in the presence of an absorbing boundary $S, y_{0}<S<B_{2}$. Then, Eq. (1) describes the dynamics of $Y_{t}$ until it crosses $S$ for the first time, the so called first-passage time (FPT), defined as

$$
T:=\inf \left\{t \geq 0: Y_{t} \geq S \mid y_{0}<S\right\},
$$

with pdf $g(t):=g\left(t \mid y_{0}, S\right)$. The moments of $T$ can be calculated through the Siegert's formula ${ }^{20}$

$$
\begin{gathered}
\mathbb{E}\left[T^{n}\right]=n \int_{x_{0}}^{S} \frac{2 d z}{\left[\Sigma\left(Y_{t}\right)\right]^{2} \mathcal{W}(z)} \int_{-\infty}^{z} \mathcal{W}(x) \mathbb{E}\left[T^{n-1}\right] d x, \\
n=1,2, \ldots
\end{gathered}
$$

Three distinct situations for the FPT can occur. The process is said to be in the suprathreshold, subthreshold, and threshold regimes if $\mathbb{E}\left[Y_{\infty}\right]>S, \mathbb{E}\left[Y_{\infty}\right]<S$, and $\mathbb{E}\left[Y_{\infty}\right]=S$, respectively.

\section{A. The Jacobi diffusion process}

The aforementioned Jacobi diffusion ${ }^{19}$ is a stochastic process with state space $(0,1)$, given as the solution to the following stochastic differential equation

$$
d Y_{t}=\left(-\alpha Y_{t}+\beta\right) d t+\sigma \sqrt{Y_{t}\left(1-Y_{t}\right)} d W_{t}, \quad Y_{0}=y_{0},
$$

with $\alpha>0, \sigma>0, y_{0} \in(0,1)$. Sometimes, it is alternatively defined on the interval $(-1,1)$ by setting $\Sigma\left(Y_{t}\right)=\sigma_{J} \sqrt{1-Y_{t}^{2}}, \sigma_{J}>0$. Throughout, we will consider the Jacobi diffusion given by (6). The conditional variance of the Jacobi process, the moment generating function, and the transition pdf are given in Refs. 18, 21, and 22, respectively.

Let $\eta:=2 \alpha / \sigma^{2}$ and $\gamma:=2 \beta / \sigma^{2}$. Then, for $\gamma>0$ such that $\min (\gamma, \eta-\gamma) \geq 1$, the boundaries 0 and 1 are of entrance type $^{22}$ and the Jacobi diffusion admits a stationary distribution $\mathcal{W}$. In particular, $\mathcal{W}$ is a beta-distribution with shape parameter $\gamma$ and scale parameter $\eta-\gamma$, i.e., ${ }^{18}$

$$
Y_{\infty} \sim \operatorname{Beta}(\gamma, \eta-\gamma),
$$

with mean (3) and variance $\operatorname{Var}\left(Y_{\infty}\right)=\beta(\alpha-\beta) \sigma^{2} /\left(2 \alpha+\sigma^{2}\right)$.

In the presence of a constant threshold $S$, with $0<y_{0}<S<1$, the Laplace transform of the FPT $T$, i.e., 
$g^{*}(\xi):=\mathbb{E}\left[e^{-\xi T}\right]=\int_{0}^{\infty} e^{-\xi t} g(t) d t, \xi>0$, is given by ${ }^{21}$

$$
g^{*}(\xi)=\frac{{ }_{2} F_{1}\left(\frac{2 \xi}{\theta \sigma^{2}}, \theta ; \gamma ; y_{0}\right)}{{ }_{2} F_{1}\left(\frac{2 \xi}{\theta \sigma^{2}}, \theta, \gamma ; S\right)},
$$

where

$$
\theta=\frac{2 \alpha-\sigma^{2}-\sqrt{\left(\sigma^{2}-2 \alpha\right)^{2}-8 \xi \sigma^{2}}}{2 \sigma^{2}} .
$$

Here, ${ }_{2} F_{1}$ denotes the Gaussian hypergeometric function belonging to the class of the generalized hypergeometric functions ${ }_{p} F_{q}$ defined by

$$
{ }_{p} F_{q}\left(a_{1}, \ldots, a_{p} ; b_{1}, \ldots, b_{q} ; z\right):=\sum_{n=0}^{\infty} \frac{\left(a_{1}\right)_{n} \cdots\left(a_{p}\right)_{n}}{\left(b_{1}\right)_{n} \cdots\left(b_{q}\right)_{n}} \frac{z^{n}}{n !},
$$

where $(a)_{n}$ is the rising factorial defined by $(a)_{n}=a(a+1)$ $\cdots(a+n-1)$ for $n \in \mathbb{N}$ and $(a)_{0}=1$. The mean

$$
\begin{aligned}
\operatorname{Var}(T)= & \frac{\mathbb{E}[T]}{\beta}\left[S_{3} F_{2}(1,1, \eta ; 2, \gamma+1 ; S)+y_{0}{ }_{3} F_{2}\left(1,1, \eta ; 2, \gamma+1 ; y_{0}\right)\right]-\frac{4}{\sigma^{2} \beta} \sum_{k=0}^{\infty} \frac{(\eta)_{k}}{(\gamma+1)_{k}(k+1)(k+2)(\gamma+k+1)} \\
& \times\left[{ }_{3} F_{2}(1, k+2, \eta+k+1 ; k+3, \gamma+k+2 ; S) S^{k+2}-{ }_{3} F_{2}\left(1, k+2, \eta+k+1 ; k+3, \gamma+k+2 ; y_{0}\right) y_{0}^{k+2}\right] . \quad(12)
\end{aligned}
$$

To evaluate Eq. (12) numerically, we replace the series with the sum of the first $k$ terms. Our comparisons for different values of $k$ with Monte Carlo simulations show that the series (and thus the value of the variance) can be satisfactorily computed by summing the first 20 terms. All the numerical results presented in the following parts were carefully verified by extensive simulations with relative difference always below 1\%. Finally, we also derive the third moment of $T$ using the Siegert formula (5), obtaining

$$
\begin{aligned}
\mathbb{E}\left[T^{3}\right]= & \frac{3 S \mathbb{E}\left[T^{2}\right]}{\beta}{ }_{3} F_{2}(1,1, \eta ; 2, \gamma+1 ; S)-12 \frac{\mathbb{E}[T]}{\sigma^{2} \beta} \sum_{k=0}^{\infty} \frac{(\eta)_{k} F_{2}(1, k+2, \eta+k+1 ; k+3, \gamma+k+2 ; S) S^{k+2}}{(\gamma+1)_{k}(k+1)(k+2)(\gamma+k+1)} \\
& +\frac{24}{\sigma^{4} \beta} \sum_{k=0}^{\infty} \frac{(\eta)_{k}}{(\gamma+1)_{k}(k+1)(\gamma+k+1)} \sum_{n=0}^{\infty} \frac{(\eta+k+1)_{n}}{(\gamma+k+2)_{n}(k+n+2)(\gamma+k+n+2)} \\
& \times\left[{ }_{3} F_{2}(1, k+n+3, \eta+k+n+2 ; k+n+4, \gamma+k+n+3 ; S) S^{k+n+3}\right. \\
& \left.-{ }_{3} F_{2}\left(1, k+n+3, \eta+k+n+2 ; k+n+4, \gamma+k+n+3 ; y_{0}\right) y_{0}^{k+n+3}\right] .
\end{aligned}
$$

The derived expressions of the first three moments can be used to perform moment estimation of the parameters $\alpha$, $\beta$, and $\sigma$ appearing in Eq. (6). This would be particularly interesting in neuroscience, where parameter estimation from FPT data has already been successfully applied for other diffusion models. ${ }^{26-29}$ Moreover, it will allow a comparison with the results available for the parameter inference on $\alpha$ and $\beta$ for the Jacobi process for continuously recorded observations of the trajectory of $X(t)$ (modeling the membrane voltage). ${ }^{30}$

In Fig. 1, we illustrate the inverse mean FPT, defined as $1 / \mathbb{E}[T]$, the coefficient of variation $(\mathrm{CV})$ of $T$, defined as the ratio between the standard deviation and the mean of $T$, i.e., $\mathrm{CV}(T)=\sqrt{\operatorname{Var}(T)} / \mathbb{E}[T]$, and the stationary distribution $\mathcal{W}$ of the Jacobi diffusion (6) given by Eq. (7). As expected, the inverse mean FPT increases and CV decreases with increasing drift coefficient, $\beta$. Further, the asymptotic distribution gets more symmetric for large values of $\beta$. Both the inverse mean FPT as well as CV increase with increasing the noise amplitude and the curves become smoother.

\section{B. The Jacobi diffusion as a neuronal model}

Equation (1) with various noisy terms is widely used in the context of neuroscience. It describes the subthreshold dynamics of the membrane voltage of a nerve cell, i.e., the evolution of the membrane depolarization between two consecutive spikes (firing), modeled as crossings of a certain threshold. In this way, the interspike interval (ISI) is identified with the FPT of the mathematical model. With $\Sigma\left(Y_{t}\right)=0$, constant absorbing threshold $S$, time constant $\tau=1 / \alpha$, and constant or time-varying input $\beta$, Eq. (1) has often been investigated as a deterministic leaky integrate-and-fire neuronal model. ${ }^{31}$ To take the underlying 

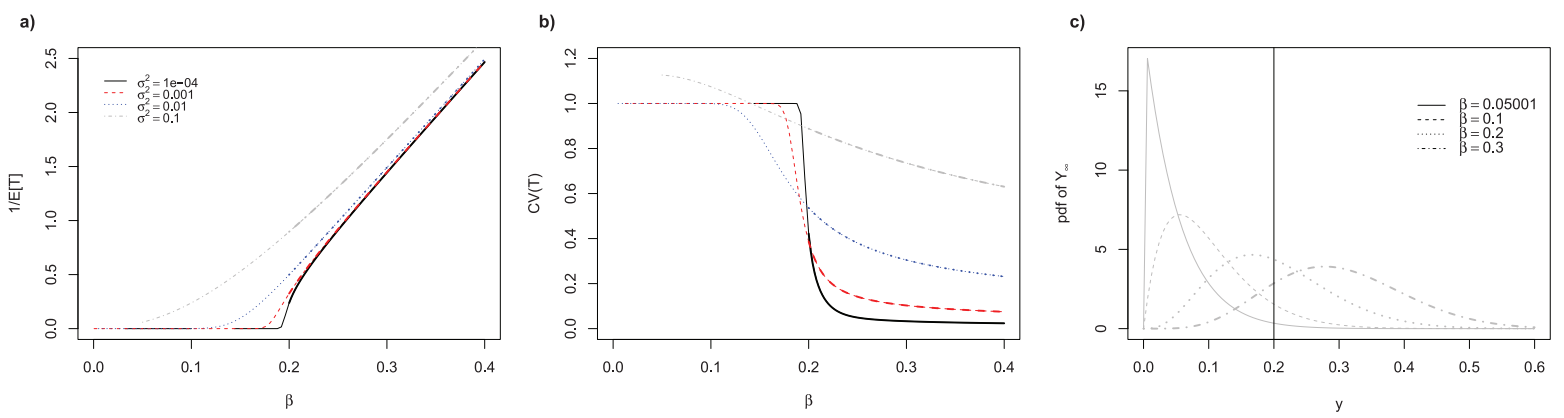

FIG. 1. Some properties of the Jacobi diffusion. (a) and (b) Inverse mean FPT and $\mathrm{CV}(T)$ as a function of the drift coefficient, $\beta$, for different values of the noise, $\sigma^{2}$. The process is in suprathreshold regime for $\beta>0.2$ (thick lines). In both figures, $y_{0}=0.1, \alpha=1, S=0.2$. (c) pdf of the asymptotic distribution $\mathcal{W}$ of $Y_{\infty}$ for different values of $\beta$ with $y_{0}=0.1, \alpha=1$, and $\sigma^{2}=0.1$. Values of $\beta \leq 0.05$ do not satisfy the condition $\min (\gamma, \eta-\gamma) \geq 1$ in this case. The vertical line represents the threshold $S$.

intrinsic neuronal randomness into account, the model was often extended by adding a white or colored noise.

The diffusion process (1) has been often obtained as a diffusion limit (also known as diffusion approximation) of discontinuous jump process under the assumption of increasing frequency and decreasing size of the jumps. ${ }^{9,11,32,33}$ An example of the initial discontinuous process is the Stein's model with reversal potentials $V_{I}$ and $V_{E}$ given by the following stochastic differential equation,

$$
\begin{aligned}
d X_{t}= & -\frac{1}{\tau} X_{t} d t+a\left(V_{E}-X_{t}\right) d N^{+}(t) \\
& +i\left(X_{t}-V_{I}\right) d N^{-}(t), \quad X_{0}=x_{0},
\end{aligned}
$$

where $i$ and $a$ are constants such that $-1<i<0<a<1$. Here, $\tau>0$ is the membrane constant taking into account the spontaneous voltage decay toward the resting potential $V_{R}$ in the absence of input, and the inhibitory and excitatory reversal potentials $V_{I}$ and $V_{E}$ are such that $V_{I}<0<V_{E}$. We assume $V_{R}=x_{0}=0$. In Eq. (13), $N^{+}:=\left\{N^{+}(t), t \geq 0\right\}$ and $N^{-}:=\left\{N^{-}(t), t \geq 0\right\}$ are two independent homogeneous Poisson processes with $N^{+}(0)=N^{-}(0)=0$ and intensities $\lambda$ and $\omega$, respectively. The processes $N^{+}$and $N^{-}$represent the excitatory and inhibitory neuronal inputs, respectively, while $\lambda$ and $\omega$ are called the excitatory and inhibitory input parameters and represent the rate of arrival of excitatory and inhibitory postsynaptic potentials. According to model (13), each event of the excitatory process $N^{+}$produces a jump in the membrane voltage $\Delta X=a\left(V_{E}-X_{t}\right)$. Similarly, an event of the inhibitory process $N^{-}$hyperpolarizes the membrane by $i\left(X_{t}-V_{I}\right)$. This implies that the effect of the input is statedependent: the amplitude of changes in the depolarization $X_{t}$ decreases when $X_{t}$ approaches the inhibitory or the excitatory reversal potentials $V_{I}$ and $V_{E}$. A direct consequence is that the process is constrained on the interval $\left(V_{I}, V_{E}\right)$, i.e., the state space of the process is $\left(V_{I}, V_{E}\right)$.

Under the assumptions of jump amplitudes $a, i$ decreasing to zero but occurring at increasing frequencies $\lambda, \omega$ roughly inversely proportional to the square of the jump size, a diffusion approximation of a generalization of Eq. (13), including random jump amplitudes depending simultaneously on both reversal potentials, was proposed. ${ }^{11}$ The stochastic differential equation describing this dynamics is

$$
\begin{aligned}
d X_{t} & =\left[-\frac{X_{t}}{\tau}+\mu\left(V_{E}-X_{t}\right)+v\left(X_{t}-V_{I}\right)\right] d t \\
& +\sigma \sqrt{\left(V_{E}-X_{t}\right)\left(X_{t}-V_{I}\right)} d W_{t}, \quad X_{0}=0,
\end{aligned}
$$

where

$$
\mu=a \lambda, \quad v=i \omega .
$$

The parameter $\sigma^{2}$ determines the amplitude of the noise, and it is often assumed to grow linearly with the input. ${ }^{34-36}$ Following this, we let the noise intensity vary according to the formula

$$
\sigma^{2}=(\lambda+\omega) \epsilon,
$$

obtained through the diffusion approximation. Here, the constant $\epsilon>0$ determines the relation between the input parameters and the noise amplitude. The asymptotic depolarization of the process $X_{t}$ is denoted by $X_{\infty}$, and its mean is given by

$$
\mathbb{E}\left[X_{\infty}\right]=\frac{\mu V_{E}-v V_{I}}{\frac{1}{\tau}+v-\mu} .
$$

For a better understanding of the parameters $\mu$ and $\nu$, we recall the equation of a conductance-based neuronal model. The evolution in time of the potential difference $V$ across the membrane of a neuron is given by (see, e.g., Refs. 14 and 37)

$$
d V_{t}=-\frac{g_{L}}{C} V_{t} d t-\left[\frac{g_{E}(t)}{C}\left(V_{t}-V_{E}\right)+\frac{g_{I}(t)}{C}\left(V_{t}-V_{I}\right)\right] d t,
$$

where $C$ is the membrane capacitance, $g_{L}$ is the conductance of the leak current, while $g_{E}(t)$ and $g_{I}(t)$ are the conductances of the excitatory and inhibitory components of the synaptic current, respectively. Equation (18) is analogous to Eq. (13) with $g_{L} / C$ playing the role of the membrane constant $\tau$ and with $g_{E}(t)=C a_{E} \sum_{k} \delta\left(t-t_{k}\right)$, where $a_{E}$ is a dimensionless constant measuring the strength of the synapse and $t_{k}$ is the time of the arrival of the $k$-th incoming excitatory pulse, distributed according to a Poisson process with parameter $\lambda$ [similarly for $g_{I}(t)$ and $\omega$ ]. The arrival of one excitatory pulse increases the conductance $g_{E}(t)$ by a factor of $C a_{E}$, and consequently, the increase in the voltage is $\Delta V=a_{E}\left(V_{E}-V_{t}\right)$. 

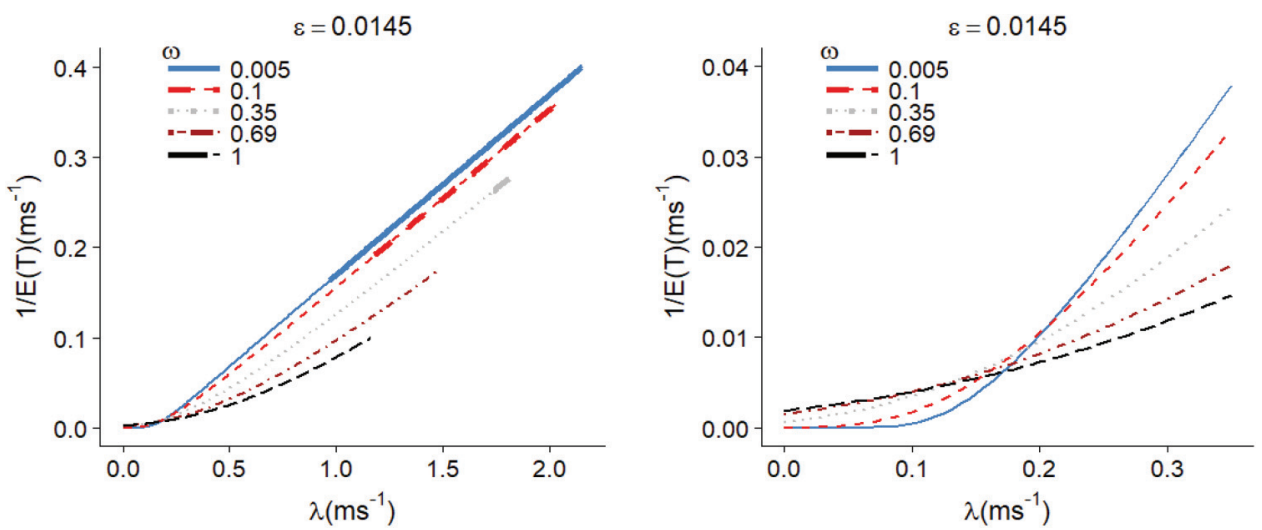

FIG. 2. Firing rate, $1 / \mathbb{E}(T)$, for the Jacobi diffusion neuronal model, as a function of the excitatory rate $\lambda$ for several fixed levels of the inhibitory rate $\omega$; the values of $\lambda$ are limited by condition (19). The right panel is a zoomed version of the left one. The same parameter values of Example $1 \mathrm{of}$ Ref. $21: V_{I}=-10 \mathrm{mV}$, $V_{E}=100 \mathrm{mV}, S=10 \mathrm{mV}, \tau=5.8 \mathrm{~ms}, i=-0.2, a=0.02, x_{0}=0 \mathrm{mV}$, and $\epsilon=0.0145$ as in Ref. 36. The values of $\omega$ are in the legend (ms ${ }^{-1}$ ). We note a divisive effect for low frequencies and subtracting effect for high frequencies of the excitatory input, as also shown in Ref. 36 . The lines become thick when the chosen parameters yield the process in the suprathreshold regime.

Focusing again on the model (14), we see that the state space of $X_{t}$ is again the interval $\left(V_{I}, V_{E}\right)$, where $V_{I}$ and $V_{E}$ are both entrance boundaries if the following condition is satisfied

$$
\epsilon(\lambda+\omega)<-\frac{2 V_{I}}{\tau\left(V_{E}-V_{I}\right)}
$$

Throughout the paper, the underlying parameters are chosen to guarantee that this boundary condition is met.

As previously mentioned, three firing regimes can be considered for the process $Y_{t}$ and thus for the process $X_{t}$. Referring to the latter model, if the asymptotic mean depolarization (17) is larger than the firing threshold $S$, then the process is in the suprathreshold regime. If it is smaller, then it is in the subthreshold regime, and the noise plays a crucial role for the crossing of the threshold. Finally, if the asymptotic mean is equal to $S$, the process is in the threshold regime. However, due to the interplay of the parameters, the specific situation is more complicated and the role played by the noise may be counterintuitive, as seen later. Using the transformation from Eq. (14) we get Eq. (6), whose solution is confined in $(0,1)$, with

$$
\begin{aligned}
\alpha & =\frac{1}{\tau}+\mu-v, \quad \beta=\mu-\frac{V_{I}}{\tau\left(V_{E}-V_{I}\right)}, \\
Y_{0} & =y_{0}=-\frac{V_{I}}{V_{E}-V_{I}} .
\end{aligned}
$$

All the analytical results previously presented for the Jacobi process in $(0,1)$ can thus be used. However, it is important to stress that the coefficients $\alpha, \beta$, and also the noise $\sigma^{2}$ are now input dependent, being functions of the input parameters $\lambda$ and $\omega$.

\section{INPUT-OUTPUT PROPERTIES OF THE JACOBI NEURONAL MODEL}

\section{A. Firing rate}

The relation between the level of synaptic input received by a neuron and the frequency of the generated action potentials (firing frequency) is commonly reflected by the so-called $f$-I curve. The activation of an inhibitory input can produce in the neuron a change in its membrane potential (hyperpolarization) and/or an increase in the membrane conductance
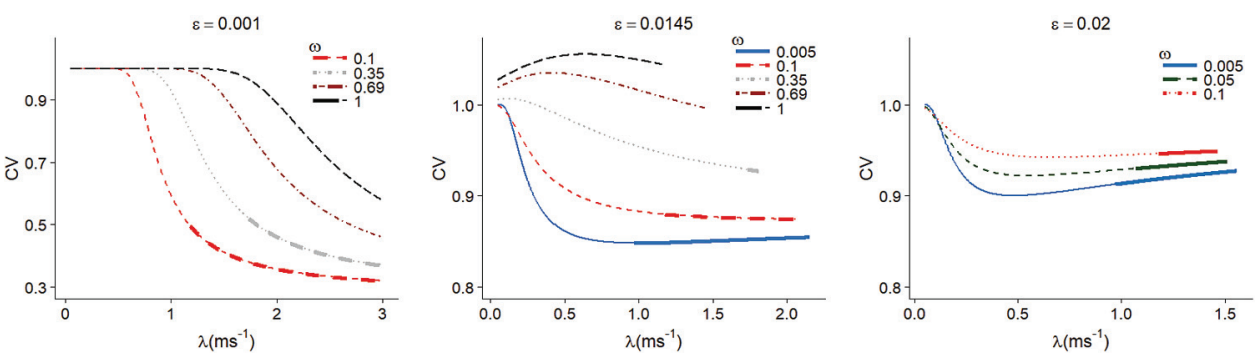

FIG. 3. Coefficient of variation of the ISIs generated by the Jacobi diffusion neuronal model as a function of the excitatory rate $\lambda$ for several choices of the inhibitory rate $\omega$. The parameters are chosen as in Fig. 2. Left figure: $\epsilon=0.001$. The CVs monotonically decrease in $\lambda$ and take small values for low inhibitory inputs. Central figure: $\epsilon=0.0145$. The CVs grow with increasing inhibition. For high values of $\omega$, the curves reach a maximum value and then change concavity. Right figure: $\epsilon=0.02$. For values of $\omega$ close to zero, the CV curves have minima. All CVs are plotted as a function of $\lambda$ such that Eq. (19) is fulfilled, and this is the reason why some lines stop before others. The lines become thick when the chosen parameters yield a process in the suprathreshold regime. All maxima and minima occur in the subthreshold regime in the considered cases. 
(shunting inhibition). ${ }^{38,39}$ The latter effect modifies the sensitivity of a neuron to a variation in the excitatory input rate ${ }^{40}$ and produces a change in the slope of the $f-I$ curve for different inhibitory input rates (divisive effect). ${ }^{36}$ If the slope is high, a small variation in the excitatory input rate produces a big difference in the resulting firing rate. The hyperpolarization decreases the membrane depolarization reducing the effect of a subsequent excitatory stimulation. In this case, the $f-I$ curve shifts for different inhibitory input rates but keeps its slope (subtractive effect). In this section, we investigate how the dependence of the coefficients $\alpha, \beta, \sigma$ in the model (14) on the input rates given by Eqs. (16) and (20) affects the FPTs (and thus the ISIs) of the Jacobi neuronal model.

As already mentioned, the firing frequency is commonly replaced by the inverse of the mean ISI, $1 / \mathbb{E}(T)$. In this way, we constructed the $f-I$ curve for model (14) considering the same physiologically realistic parameters as those given in Example 1 of Ref. 21: $V_{I}=-10 \mathrm{mV}, V_{E}=100 \mathrm{mV}$, $S=10 \mathrm{mV}, \tau=5.8 \mathrm{~ms}, i=-0.2, a=0.02, x_{0}=0 \mathrm{mV}$, for different values of $\epsilon, \lambda$, and $\omega$, namely, $\lambda \in(0,3), \omega \in(0,1)$, and $\epsilon=0.001,0.0145$ (as in Ref. 36), and 0.02.

In Fig. 2, we plot the firing rate as a function of the excitatory rate $\lambda$ for different values of the inhibitory rate $\omega$ and observe a divisive effect of inhibition for low frequencies of the excitatory input and a subtracting effect for high frequencies. For higher values of $\lambda$, the deterministic force characterized by $\beta$ drives the neuron to fire. The behavior of $1 / \mathbb{E}(T)$ for the neuronal model (14) differs from that of the general Jacobi diffusion (6) reported in Fig. 1. There, the inverse mean FPT increases when $\sigma^{2}$ increases, while here, the firing rate decreases in the suprathreshold regime if we increase $\sigma^{2}$ by increasing the inhibitory rate, $\omega$ [cf. Eq. (16)].

If we decrease the value of the reversal potential $V_{E}$, the excitatory term $\mu\left(V_{E}-X_{t}\right)$ in (14) has a weakened effect (figure not shown). The $f-I$ curve shifts to the right for increasing inhibition $\omega$ compared to the case of Fig. 2. This is because changing the reversal potential is equivalent to change the input by a constant quantity. ${ }^{41}$ Also, in this case, divisive and subtractive effects are observed. Thus, their presence does not depend on the value of the reversal potential.

\section{B. Firing variability}

Using Eqs. (11) and (12), in Fig. 3, we plot the CV of the ISIs generated by model (14) as a function of the excitatory rate $\lambda$ for different fixed values of the inhibitory rate $\omega$. In all the considered cases, the CV grows with increasing inhibition. For $\epsilon=0.001$, it means very small level of noise; as seen from Eq. (16), the CVs are smaller than one and are decreasing functions of the excitatory rate $\lambda$. For bigger $\epsilon$, we observe values of the $\mathrm{CV}$ greater than one and different behaviors, concavities, and types of monotonicity of the CV for different values of $\omega$. Interestingly, the CVs are non-monotonic in $\lambda$, and some CVs exhibit a maximum or a minimum for certain values of the input parameters. Note that, in the cases considered here, all maxima and minima occur in the subthreshold regime (the thick lines in the figure indicate the suprathreshold regime). Many of these features are not present in the CVs of the Jacobi diffusion (6) reported in Fig. 1, suggesting a qualitative difference between the general and the neuronal-based models.

To illustrate the simultaneous dependence of the $\mathrm{CV}$ on the inhibitory and the excitatory inputs, we report its heat map in Fig. 4 (left) for $\epsilon=0.0145$. All CVs increase in $\omega$; CVs larger than one can be obtained by either increasing the inhibitory inputs or decreasing the excitatory inputs, while CVs smaller than one are only observed for low values of $\omega$. Interestingly, for a fixed inhibitory input $\omega$, it is possible to decrease the CVs by either increasing the excitatory input (as one would expect) or decreasing it. The blue lines report the contour plots of the CVs, i.e., the values of $(\lambda, \omega)$ yielding the same CVs, while the black dots represent the values of $\lambda$, denoted by $\lambda^{*}$, maximizing the $\mathrm{CV}$ for a fixed $\omega$. The values of $\lambda^{*}$ increase with $\omega$, suggesting some relation in the proportion
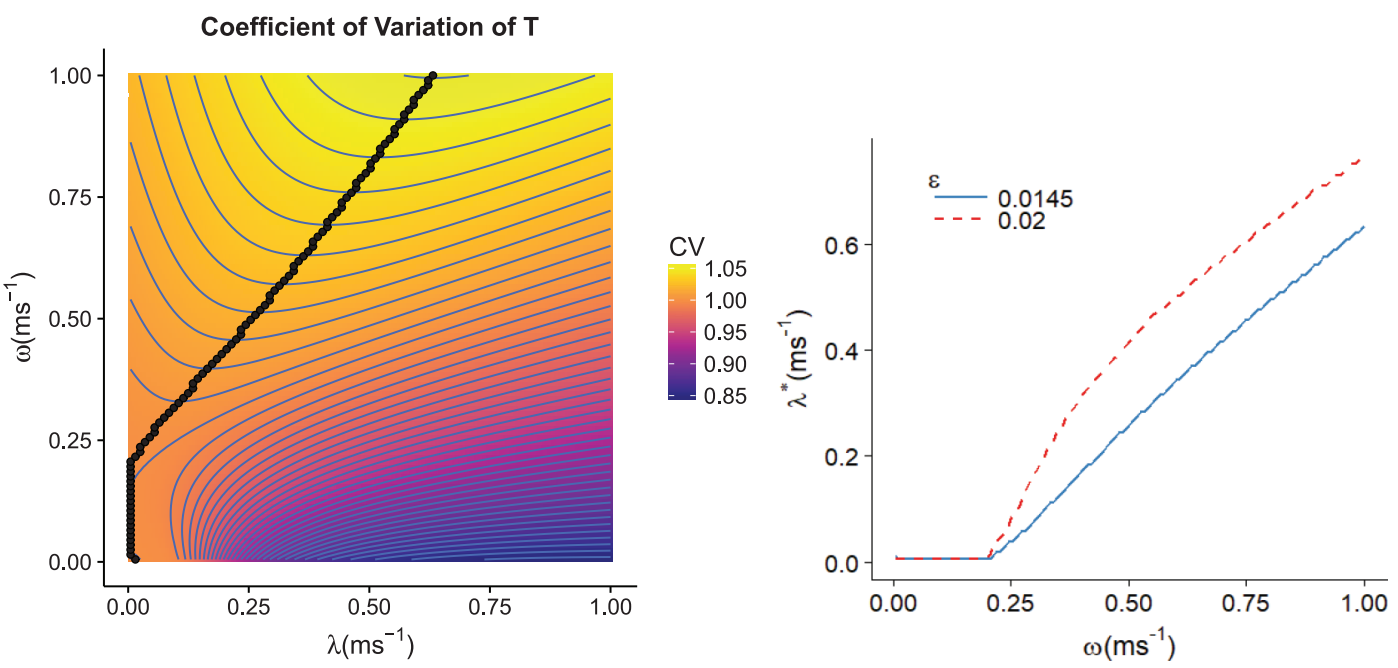

FIG. 4. Left figure: Heat map of the dependency of the CV of $T$ on both inhibitory and excitatory inputs. The ranges of values of $\lambda$ and $\omega$ are written on the axes labels; the other parameters are the same as Fig. 2. The yellowish areas represent values of the CV larger than one. The blue lines are the curves along which the $\mathrm{CV}$ has a constant value, i.e., the $2 \mathrm{D}$ sections of the $3 \mathrm{D}$ plot (contour lines). The black points are $\left(\lambda^{*}, \omega\right)$, where $\lambda^{*}$ denotes the value of $\lambda$ maximizing the $\mathrm{CV}$ of $T$ for a given $\omega$. Right figure: Relationship between $\omega$ and $\lambda^{*}$ for two different values of $\epsilon$. Note that $\lambda^{*}$ increases for increasing absolute values of $\omega$; however, the relation is not always linear as it may look from the heat map. 
of inhibitory and excitatory inputs. This can be observed in Fig. 4 (right), where we report $\left(\omega, \lambda^{*}\right)$ for two values of $\epsilon$.

\section{The role of input-dependent parameters}

What is the reason causing the different behaviors of the inverse mean FPT (firing rate) and the $\mathrm{CV}(T)$ for the Jacobi diffusion (6) and the neuronal model (14)? To answer this question, we investigate the role played by the inhibitory and excitatory inputs $\omega$ and $\lambda$ in the coefficients $\alpha, \beta$, and $\sigma^{2}$ of the neuronal model (14). In the general Jacobi model (6), if seen as a neuronal model, the only variable coefficient is $\beta$, while in (14), the inputs affect all three coefficients. To answer the question posed above, keeping the notation of Sec. II B, we study how $1 / \mathbb{E}(T)$ and $\mathrm{CV}(T)$ change if the input parameters act only on two of the three coefficients.

In the first row of Fig. 5, we fix $\alpha=1 / \tau$ constant, i.e., independent of the input parameters. Under this scenario, we observe, except for very low values of $\omega$, a small subtractive effect in the $f-I$ curve for low values of $\lambda$. Almost no
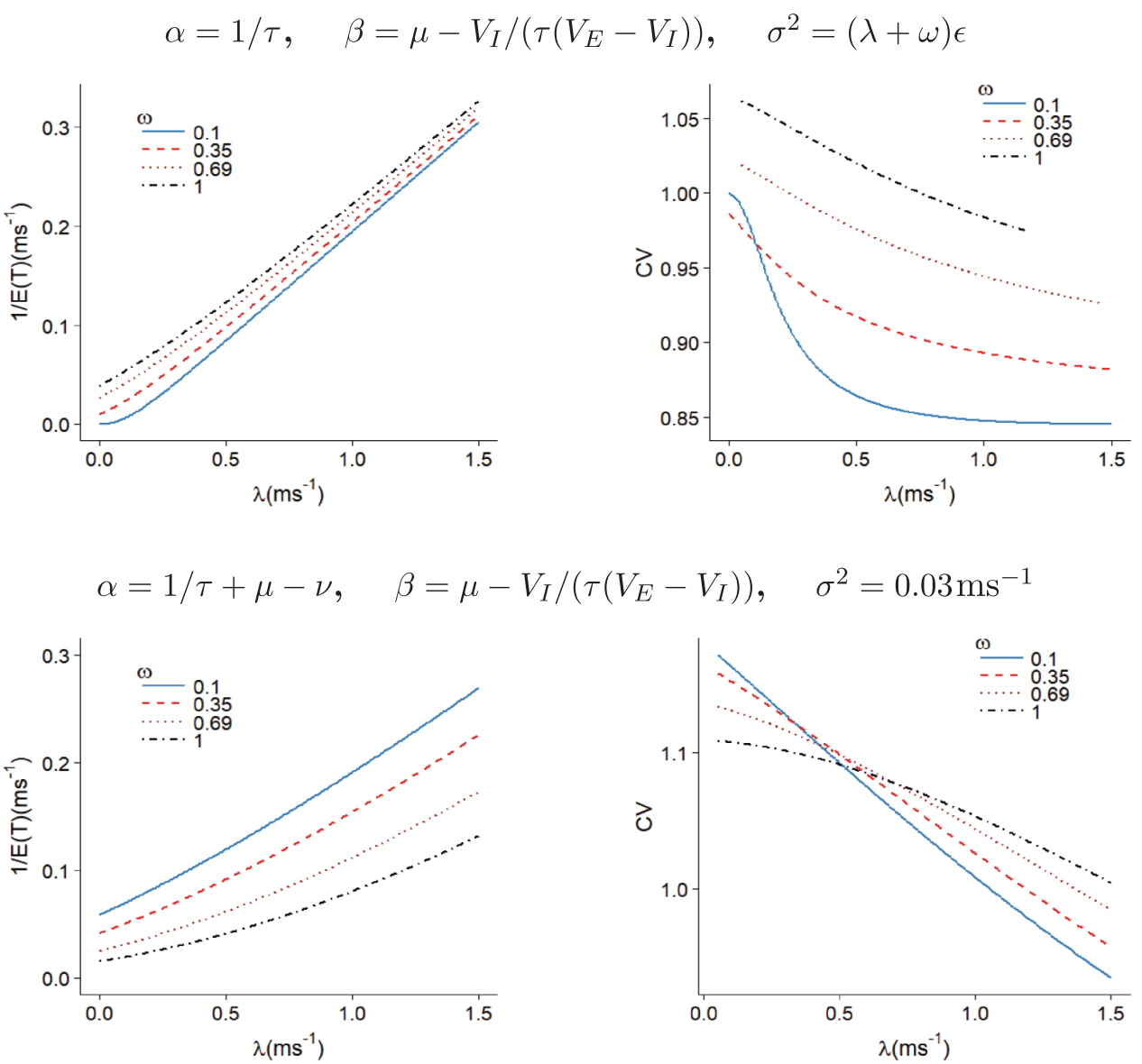

$-V_{I} /\left(\tau\left(V_{E}-V_{I}\right)\right), \quad \sigma^{2}=0.03 \mathrm{~ms}^{-1}$
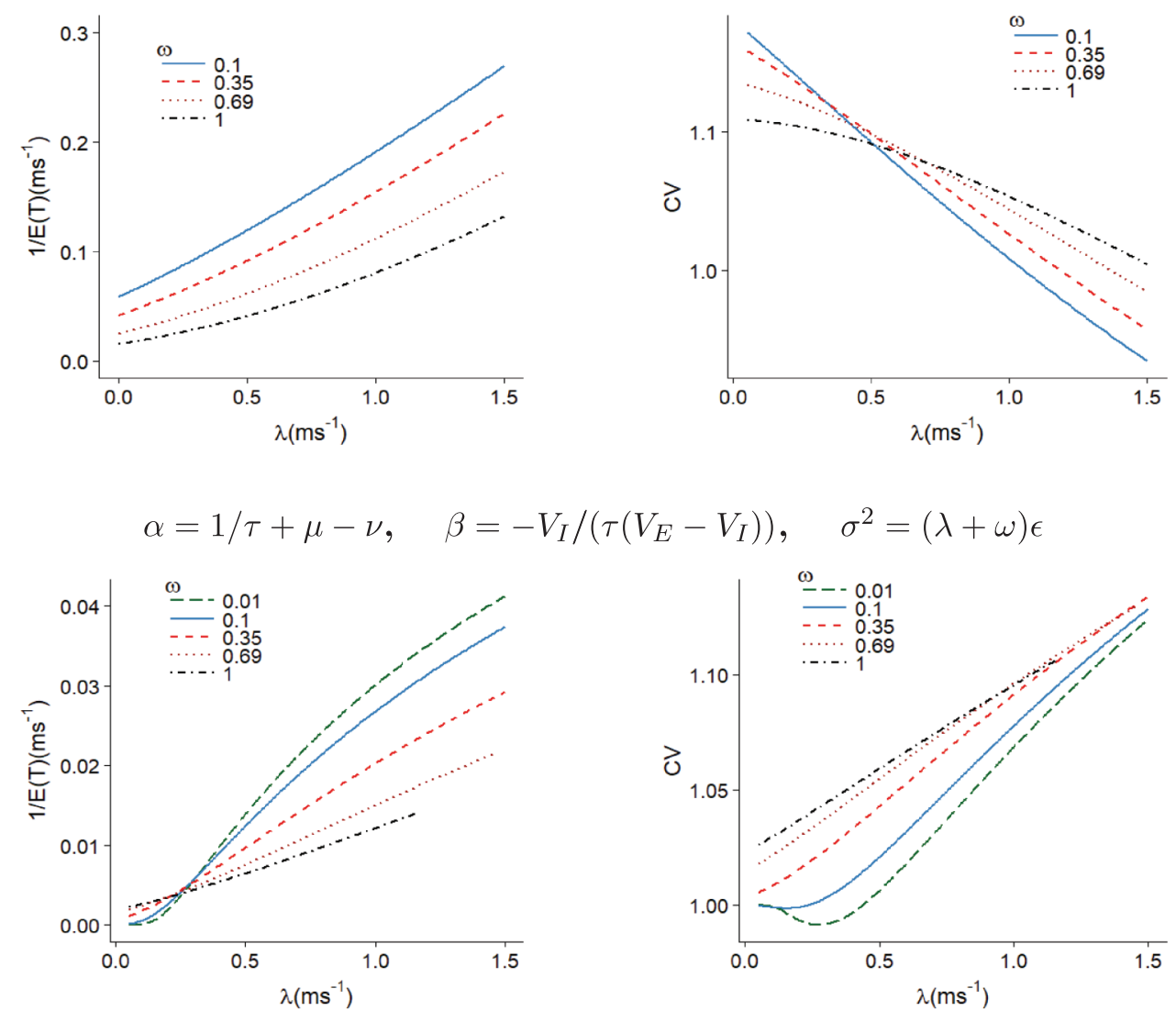

FIG. 5. Firing rate, $1 / \mathbb{E}(T)$, and coefficient of variation of the ISIs generated by the Jacobi diffusion neuronal model as a function of the excitatory rate $\lambda$ for several choices of the inhibitory rate $\omega$ if the input parameters act on two of the three coefficients $\alpha, \beta$, and $\sigma^{2}$ only. In the first row, $\alpha=1 / \tau$ constant (i.e., independent of the input parameters $\lambda$ and $\omega$ ); in the second row, $\sigma^{2}=0.03 \mathrm{~ms}^{-1}$ constant; and in the third row, $\beta=-V_{I} /\left[\tau\left(V_{E}-V_{I}\right)\right]$ while $\alpha$ and $\sigma^{2}$ change with $\lambda$ and $\omega$. We recall from Eq. (15) that $\mu=a \lambda$ and $v=i \omega$. 
effect is seen for high values of $\lambda$. It suggests that the noise alone cannot produce large divisive effects. Similarly, no divisive effects are observed when $\alpha=1 / \tau+\mu$, i.e., when $\alpha$ depends on the excitation only $(\mu=a \lambda)$ but not on the inhibition (results not shown). In contrast to Fig. 2, the firing rate increases with the inhibition in this case. This is because the parameter $\omega$ effects $\sigma^{2}$ only and in this way increases the fluctuations of the membrane depolarization and thus the firing rate. On the right panel of the first row of Fig. 5, one can see that the CV increases when the rate of inhibitory input, $\omega$, increases and is monotonically decreasing function of $\lambda$. These results agree qualitatively with what we observed in Fig. 1 for the Jacobi diffusion (6).

In the second row of Fig. 5, we let $\alpha$ and $\beta$ vary with the input parameters and fix $\sigma^{2}=0.33 \mathrm{~ms}^{-1}$, physiological value used in Ref. 21. A divisive effect is not visible in the $f-I$ curve, while the subtractive one is strong. In this scenario, the firing rate decreases if the inhibitory rate increases. The CVs are monotonically decreasing functions of $\lambda$ that intersect for different values of $\omega$. For $\lambda$ and $\omega$ close to zero, the $\mathrm{CV}$ is greater than one because, differently from before, $\sigma^{2}$ does not depend on them and therefore, it does not tend to zero. Interestingly, increasing the inhibition reduces the CVs for values of $\lambda$ smaller than $0.5 \mathrm{~ms}^{-1}$.

In the third row of Fig. 5, we consider $\beta=-V_{I} /$ $\left[\tau\left(V_{E}-V_{I}\right)\right]$ independent of $\lambda$, while $\alpha$ and $\sigma^{2}$ are varying with the input parameters. Both the divisive and the subtractive effects are visible in the $f-I$ curve. However, the firing frequency, $1 / \mathbb{E}(T)$, is lower than before (about $1 / 10$ ). Considering the dependency of $\mathrm{CV}$ on the input, we see that for values of $\omega$ greater than $0.1 \mathrm{~ms}^{-1}$, the $\mathrm{CV}$ is monotonically increasing in $\lambda$ and larger than one. This happens because $\beta$ is constant while $\sigma^{2}$ increases in $\lambda$ causing the variability to increase. Finally, under this scenario, for small values of $\omega$ (see the case $\omega=0.01 \mathrm{~ms}^{-1}$ ), the CV curve shows a minimum, Fig. 3 (right panel). To get maxima in the CV as a function of $\lambda$, it seems that the input parameters have to act simultaneously on all the three coefficients as happens for the Jacobi neuronal model (14) with coefficients given by Eq. (20).

\section{DISCUSSION}

\section{A. Firing rate}

An increase of the firing frequency with increasing level of inhibition is a counterintuitive phenomenon. It was observed for neuronal networks, e.g., in Ref. 42, and for single neurons, e.g., in Ref. 43. Here, for low values of the excitatory rate $\lambda$, we see on the right panel of Fig. 2 that increasing the inhibition increases the firing rate. The reason is the following: if the input frequency is low (small $\lambda$ ), then the inhibition helps the firing since it increases the noise, cf. Eq. (16).

The noise alone causes only a modest divisive effect on the firing rate during shunting inhibition. Holt and $\mathrm{Koch}^{41}$ showed that for an integrate-and-fire model in the suprathreshold regime, the effect of a voltage-independent inhibition is subtractive. Doiron et al. ${ }^{44}$ extended this result to the voltagedependent case, observing a divisive effect at low frequencies of excitation for a stochastic leaky integrate-and-fire model with additive noise, provided that the noise increases with the inhibitory input. ${ }^{36}$ Divisive modulation of the firing rate under noisy conditions is instead enhanced by a nonlinear integration of the inputs. ${ }^{45}$ In fact, it is known that dendritic integration of excitatory and inhibitory inputs can be highly nonlinear due to the dendritic saturation. ${ }^{46}$ In Ref. 47, the authors proposed a dendritic integrate-and-fire model incorporating the nonlinearity of the dendritic integration rule, at the expense of a heavier mathematical formulation. The synaptic inputs are summed linearly in the original Stein's model, ${ }^{5}$ but the inclusion of the reversal potentials introduces a nonlinear synaptic summation. Here, we argue that in neuronal models with multiplicative noise, like the Jacobi diffusion considered in Eq. (6) with parameters given by Eq. (20), a fundamental role to obtain divisive phenomena is played by the coefficient $\alpha$ and in particular its dependence on the underlying inhibitory inputs. We speculate that not only the role of the noise is crucial, ${ }^{48}$ but also $1 / \alpha$ has to decrease for increasing $\omega$ to obtain a divisive effect (see the third row of Fig. 5).

\section{B. Variability of the response}

Traditionally, the neural code is assumed to be hidden in the firing frequency. Nevertheless, recently, the spiking variability attracts attention of neuroscientists as an alternative or at least as an auxiliary part of the code. ${ }^{49-51}$ Two interchangeable measures of variability, the Fano factor and the coefficient of variation, have been employed. ${ }^{52-54}$ Here, due to the knowledge of the first two moments of the ISIs, we evaluated the $\mathrm{CV}$ as a function of the excitation rate. It is drastically affected by the dependence of the coefficients $\alpha, \beta$, and $\sigma^{2}$ on the input parameters in Eq. (20) producing three main features: CV greater than one and the presence of maxima and minima with respect to the rate of excitatory inputs, $\lambda$.

We observe minima of $\mathrm{CV}$ for small values of the inhibitory rate and for strong dependency of the noise on the input (large values of $\epsilon$ ). If the rate of inhibitory inputs, $\omega$, is small, then an increase of $\lambda$ implies a decrease in the $\mathrm{CV}$, as it happens for the general Jacobi process with fixed $\alpha$ and $\sigma^{2}$. However, if the rate of excitatory inputs, $\lambda$, is large, the noisy term $\sigma^{2}=(\lambda+\omega) \epsilon \approx \lambda \epsilon$ becomes dominant and the $\mathrm{CV}$ increases. Minima in the CV as a function of the noise were observed for the Ornstein-Uhlenbeck neuronal model..$^{55}$

In the Jacobi neuronal model with coefficients given by Eq. (20), the increase of inhibitory and excitatory rates leads to an increase of the noise $\sigma^{2}$, but at the same time to a decrease in $1 / \alpha$ that reduces the voltage fluctuations. This suppression of fluctuations was also observed in the conductance-based model ${ }^{14}$ and in vivo recordings. ${ }^{56}$ This mechanism explains the presence of maxima in Fig. 3: the $\mathrm{CV}$ grows with the noise, but then it is slowed down by $\alpha$. The maxima are not observed for $\epsilon=0.001$, because in that case, the noise is too small to produce an increase in the $\mathrm{CV}$. To the best of our knowledge, maxima in the CV for the Ornstein-Uhlenbeck neuronal model were observed only when a refractory period was added to the dynamics. ${ }^{55}$

Values of the CV larger than one are commonly found in experimental data, and investigation of neuronal models in this direction has a long history. ${ }^{7,8}$ Generally, it is perceived 
as connected to an increase of inhibition. Also, for the Jacobi neuronal model with coefficients (20), the inhibition increases the CV. In fact, even if the inhibition causes an increase of the firing rate (and thus decreases the mean of $T$ ), it increases the variance of $T$ even more substantially, producing thus a higher CV. Some authors connect large values of the CV with a balance between excitation and inhibition. For example, a highly irregular firing activity is produced in the presence of an appropriate balance of the excitatory and inhibitory input (see, e.g., Refs. 49 and 57). Christodoulou and Bugmann ${ }^{58,59}$ obtained CVs greater than one for strong inhibition, in particular for a level of inhibition that is greater than $80 \%$ of the level of excitation. Konig et al. ${ }^{60}$ argued that an exact balance (100\%) of excitatory and inhibitory inputs cannot be realistic from a biological point of view. For the Jacobi neuronal model, we observe CVs greater than one also for the inhibitory rate that is less than $50 \%$ of the rate of the excitatory one; see, for example, Fig. 3 (central panel).

For all these reasons, the Jacobi neuronal model given by Eqs. (14), (16), and (20) constitutes a good compromise between the realistic description of a neuron and the mathematical tractability. Indeed, it is able to reproduce high degree of irregularity of the real neuronal firing. A deeper understanding of the meaning and consequences of minima and maxima in the CV of the ISIs is also of interest, and it will be the object of a future work in the context of information transmission and coherence resonance.

\section{ACKNOWLEDGMENTS}

This work was supported by the Joint Research Project between Austria and the Czech Republic through Grant No. 7AMB17AT048, by the Institute of Physiology (No. RVO:67985823), and by the Czech Science Foundation project (No. 17-06943S).

${ }^{1}$ N. Brunel and M. C. W. van Rossum, "Lapicque's 1907 paper: From frogs to integrate-and-fire," Biol. Cybern. 97, 337-339 (2007).

${ }^{2}$ N. Brunel and M. C. W. van Rossum, "Quantitative investigations of electrical nerve excitation treated as polarization," Biol. Cybern. 97, 341-349 (2007).

${ }^{3}$ R. Jolivet, T. Lewis, and W. Gerstner, "Generalized integrate-and-fire models of neural activity approximate spike trains of a detailed model to a high degree of accuracy," J. Neurophysiol. 92, 959-976 (2004).

${ }^{4}$ W. Kistler, W. Gerstner, and J. vanHemmen, "Reduction of the HodgkinHuxley equations to a single-variable threshold model," Neural Comput. 9, 1015-1045 (1997).

${ }^{5}$ R. Stein, "Some models of neuronal variability," Biophys. J. 7, 37-68 (1967).

${ }^{6}$ P. I. M. Johannesma, "Diffusion models for the stochastic activity of neurons," in Neural Networks: Proceedings of the School on Neural Networks-June 1967 in Ravello, edited by E. R. Caianiello (Springer, Berlin, 1968), pp. 116-144.

${ }^{7}$ H. C. Tuckwell, "Synaptic transmission in a model for stochastic neural activity," J. Theor. Biol. 77, 65-81 (1979).

${ }^{8}$ W. J. Wilbur and J. Rinzel, "A theoretical basis for large coefficient of variation and bimodality in neuronal interspike interval distributions," $\mathrm{J}$. Theor. Biol. 105, 345-368 (1983).

${ }^{9}$ F. B. Hanson and H. C. Tuckwell, "Diffusion approximation for neuronal activity including reversal potentials," J. Theor. Neurobiol. 2, 127-153 (1983), see http://homepages.math.uic.edu/ hanson/pub/JTN83/ HansonTuckwell1983Vol2No2pp127-153.pdf.

${ }^{10}$ C. E. Smith and M. V. Smith, "Moments of voltage trajectories for Stein's model with synaptic reversal potentials,"J. Theor. Neurobiol. 3, 167-177 (1984), see https://pdfs.semanticscholar.org/5e2f/ b7bd262c407205d2814dad7b901f429c0de8.pdf.
${ }^{11}$ P. Lansky and V. Lanska, "Diffusion approximation of the neuronal model with synaptic reversal potentials," Biol. Cybern. 56, 19-26 (1987).

${ }^{12}$ G. D'Onofrio, P. Lansky, and E. Pirozzi, "On two diffusion neuronal models with multiplicative noise: The mean first-passage time properties," Chaos: An Interdiscipl. J. Nonlinear Sci. 28, 043103 (2018).

${ }^{13}$ M. Musila and P. Lansky, "On the interspike intervals calculated from diffusion approximations of Stein's neuronal model with reversal potentials," J. Theor. Biol. 171, 225-232 (1994).

${ }^{14} \mathrm{M}$. J. E. Richardson, "Effects of synaptic conductance on the voltage distribution and firing rate of spiking neurons," Phys. Rev. E 69, 051918 (2004).

${ }^{15}$ M. J. E. Richardson and W. Gerstner, "Statistics of subthreshold neuronal voltage fluctuations due to conductance-based synaptic shot noise," Chaos: An Interdiscipl. J. Nonlinear Sci. 16, 026106 (2006).

${ }^{16} \mathrm{~J}$. Cupera, "Diffusion approximation of neuronal models revisited," Math. Biosci. Eng. 11, 11 (2014).

${ }^{17}$ B. Øksendal, Stochastic Differential Equations: An Introduction with Applications, Hochschultext/Universitext (Springer, 1998).

${ }^{18}$ J. Forman and M. Sørensen., "The Pearson diffusions: A class of statistically tractable diffusion processes," Scand. J. Stat. 35, 438-465 (2008).

${ }^{19} \mathrm{~S}$. Karlin and H. Taylor, , A Second Course in Stochastic Processes (Academic Press, 1981). Vol. 2.

${ }^{20}$ A. J. F. Siegert, "On the first passage time probability problem," Phys. Rev. 81, 617-623 (1951).

${ }^{21}$ V. Lanska, P. Lansky, and C. E. Smith, "Synaptic transmission in a diffusion model for neural activity," J. Theor. Biol. 166, 393-406 (1994).

${ }^{22}$ N. Goel and N. Richter-Dyn, Stochastic Models in Biology (Academic Press, 1974).

${ }^{23}$ R. K. S. Hankin, hypergeo: The Gauss Hypergeometric Function, R package version 1.2-13 (2016).

${ }^{24} \mathrm{R}$ Core Team, $R$ : A Language and Environment for Statistical Computing (R Foundation for Statistical Computing, Vienna, 2013).

${ }^{25} \mathrm{D}$. Veestraeten, "A recursion formula for the moments of the first passage time of the Ornstein-Uhlenbeck process," J. Appl. Probab. 52, 595-601 (2015).

${ }^{26}$ J. Inoue, S. Sato, and L. M. Ricciardi, "On the parameter estimation for diffusion models of single neuron's activities,” Biol. Cybern. 73, 209-221 (1995).

${ }^{27}$ S. Shinomoto, Y. Sakai, and S. Funahashi, "The Ornstein-Uhlenbeck process does not reproduce spiking statistics of neurons in prefrontal cortex," Neural Comput. 11, 935-951 (1999).

${ }^{28}$ S. Ditlevsen and P. Lansky, "Parameters of stochastic diffusion processes estimated from observations of first-hitting times: Application to the leaky integrate-and-fire neuronal model,” Phys. Rev. E 76, 041906 (2007).

${ }^{29}$ Q. Qin, J. Wang, H. Yu, B. Deng, and W. Chan, "Reconstruction of neuronal input through modeling single-neuron dynamics and computations," Chaos: An Interdiscipl. J. Nonlinear Sci. 26, 063121 (2016).

${ }^{30} \mathrm{~V}$. Lanska and P. Lansky, "Input parameters in a one-dimensional neuronal model with reversal potentials," Biosystems 48, 123-129 (1998).

${ }^{31} \mathrm{H}$. Tuckwell, Introduction to Theoretical Neurobiology: Volume 2, Nonlinear and Stochastic Theories (Cambridge University Press, 2005).

${ }^{32} \mathrm{~L}$. Wolff and B. Lindner, "Method to calculate the moments of the membrane voltage in a model neuron driven by multiplicative filtered shot noise," Phys. Rev. E 77, 041913 (2008).

${ }^{33}$ M. Tamborrino, L. Sacerdote, and M. Jacobsen, "Weak convergence of marked point processes generated by crossings of multivariate jump processes. Applications to neural network modeling," Phys. D: Nonlinear Phenom. 288, 45-52 (2014).

${ }^{34}$ G. A. Cecchi, M. Sigman, J.-M. Alonso, L. Martínez, D. R. Chialvo, and M. O. Magnasco, "Noise in neurons is message dependent," Proc. Natl. Acad. Sci. 97, 5557-5561 (2000).

${ }^{35}$ P. Lansky and L. Sacerdote, "The Ornstein-Uhlenbeck neuronal model with signal-dependent noise," Phys. Lett. A 285, 132-140 (2001).

${ }^{36}$ A. Longtin, B. Doiron, and A. R. Bulsara, "Noise-induced divisive gain control in neuron models," Biosystems 67, 147-156 (2002).

${ }^{37}$ S. Koyama and R. Kobayashi, "Fluctuation scaling in neural spike trains," Math. Biosci. Eng. 13, 537 (2016).

${ }^{38}$ A. Destexhe, M. Rudolph, and D. Paré, "The high-conductance state of neocortical neurons in vivo," Nat. Rev. Neurosci. 4, 739-51 (2003).

${ }^{39} \mathrm{~W}$. Paulus and J. C. Rothwell, "Membrane resistance and shunting inhibition: Where biophysics meets state-dependent human neurophysiology," J. Physiol. (Lond.) 594, 2719-2728.

${ }^{40}$ S. J. Mitchell and R. Silver, "Shunting inhibition modulates neuronal gain during synaptic excitation," Neuron 38, 433-445 (2003). 
${ }^{41}$ G. R. Holt and C. Koch, "Shunting inhibition does not have a divisive effect on firing rates," Neural. Comput. 9, 1001-1013 (1997).

${ }^{42}$ M. V. Tsodyks, W. E. Skaggs, T. J. Sejnowski, and B. L. McNaughton, "Paradoxical effects of external modulation of inhibitory interneurons," J. Neurosci. 17, 4382-4388 (1997).

${ }^{43}$ J. Feng and G. Wei, "Increasing inhibitory input increases neuronal firing rate: Why and when? Diffusion process cases," J. Phys. A: Math. Gen. 34, 7493 (2001).

${ }^{44}$ B. Doiron, A. Longtin, N. Berman, and L. Maler, "Subtractive and divisive inhibition: Effect of voltage-dependent inhibitory conductances and noise," Neural. Comput. 13, 227-248 (2001).

${ }^{45}$ S. A. Prescott, Y. De Koninck, "Gain control of firing rate by shunting inhibition: Roles of synaptic noise and dendritic saturation," Proc. Natl. Acad. Sci. 100, 2076-2081 (2003).

${ }^{46}$ D. Zhang, Y. Li, M. Rasch, and S. Wu, "Nonlinear multiplicative dendritic integration in neuron and network models," Front. Comput. Neurosci. 7 56 (2013).

${ }^{47}$ D. Zhou, S. Li, X.-h. Zhang, and D. Cai, "Phenomenological incorporation of nonlinear dendritic integration using integrate-and-fire neuronal frameworks," PLoS ONE 8, 1-12 (2013).

${ }^{48}$ F. S. Chance, L. Abbott, and A. D. Reyes, "Gain modulation from background synaptic input," Neuron 35, 773-782 (2002).

${ }^{49}$ M. N. Shadlen and W. T. Newsome, "Noise, neural codes and cortical organization," Curr. Opin. Neurobiol. 4, 569-579 (1994).

${ }^{50}$ L. Kostal, P. Lansky, and J.-P. Rospars, "Neuronal coding and spiking randomness," Eur. J. Neurosci. 26, 2693-2701 (2007).

${ }^{51}$ A. K. Churchland, R. Kiani, R. Chaudhuri, X.-J. Wang, A. Pouget, and M. Shadlen, "Variance as a signature of neural computations during decision making," Neuron 69, 818-831 (2011).
${ }^{52}$ J. W. Shuai, S. Zeng, and P. Jung, "Coherence resonance: On the use and abuse of the Fano factor," Fluctuation Noise Lett. 02, L139-L146 (2002).

${ }^{53}$ M. P. Nawrot, "Analysis and interpretation of interval and count variability in neural spike trains," in Analysis of Parallel Spike Trains, edited by S. Grün and S. Rotter (Springer US, Boston, MA, 2010), pp. 37-58.

${ }^{54}$ K. Rajdl, P. Lansky, and L. Kostal, "Entropy factor for randomness quantification in neuronal data," Neural. Netw. 95, 57-65 (2017).

${ }^{55}$ B. Lindner, L. Schimansky-Geier, and A. Longtin, "Maximizing spike train coherence or incoherence in the leaky integrate-and-fire model," Phys. Rev. E 66, 031916 (2002).

${ }^{56}$ C. Monier, F. Chavane, P. Baudot, L. J. Graham, and Y. Fregnac, "Orientation and direction selectivity of synaptic inputs in visual cortical neurons," Neuron 37, 663-680 (2003).

${ }^{57}$ J. Feng and D. Brown, "Coefficient of variation of interspike intervals greater than 0.5. How and when?," Biol. Cybern. 80, 291-297 (1999).

${ }^{58}$ C. Christodoulou and G. Bugmann, "Near Poisson-type firing produced by concurrent excitation and inhibition," Biosystems 58, 41-48 (2000).

${ }^{59}$ C. Christodoulou and G. Bugmann, "Coefficient of variation vs. mean interspike interval curves: What do they tell us about the brain?," Neurocomputing 38-40, 1141-1149 (2001). . computational Neuroscience: Trends in Research 2001.

${ }^{60}$ P. Konig, A. K. Engel, and W. Singer, "Integrator or coincidence detector? The role of the cortical neuron revisited," Trends. Neurosci. 19, 130-137 (1996). 